\title{
An accurate, high-speed, portable bifunctional electrical detector for COVID-19
}

\author{
Guojun $\mathrm{Ke}^{1,2 \dagger}$, Dingkai $\mathrm{Su}^{2 \dagger}, \mathrm{Yu} \mathrm{Li}^{2 \dagger}$, Yu Zhao ${ }^{3}$, Honggang Wang ${ }^{3}$, Wanjian $\mathrm{Liu}^{4}$, Man $\mathrm{Li}^{5}$, \\ Zhiting Yang ${ }^{4}$, Fang Xiao ${ }^{6}$, Yao Yuan ${ }^{7}$, Fei Huang ${ }^{1^{*}}$, Fanyang $\mathrm{Mo}^{3^{*}}$, Peng Wang ${ }^{5^{*}}$ and \\ Xuefeng Guo ${ }^{2 *}$
}

\begin{abstract}
Coronavirus disease 2019 (COVID-19), caused by SARS-CoV-2, has rapidly spread and caused a severe global pandemic. Because no specific drugs are available for COVID19 and few vaccines are available for SARS-CoV-2, accurate and rapid diagnosis of COVID-19 has been the most crucial measure to control this pandemic. Here, we developed a portable bifunctional electrical detector based on graphene fieldeffect transistors for SARS-CoV-2 through either nucleic acid hybridization or antigen-antibody protein interaction, with ultra-low limits of detection of $\sim 0.1$ and $\sim 1 \mathrm{fg} \mathrm{mL}^{-1}$ in phosphate buffer saline, respectively. We validated our method by assessment of RNA extracts from the oropharyngeal swabs of ten COVID-19 patients and eight healthy subjects, and the IgM/IgG antibodies from serum specimens of six COVID-19 patients and three healthy subjects. Here we show that the diagnostic results are in excellent agreement with the findings of polymerase chain reaction-based optical methods; they also exhibit rapid detection speed ( $\sim 10 \mathrm{~min}$ for nucleic acid detection and $\sim 5 \mathrm{~min}$ for immunoassay). Therefore, our assay provides an efficient, accurate tool for high-throughput pointof-care testing.
\end{abstract}

Keywords: COVID-19, biosensor, nucleic acid detection, immunoassay, point-of-care testing

\section{INTRODUCTION}

In the past 20 years, humans have suffered several serious epidemics from emerging viruses, such as SARS, swine flu, Ebola, MERS and (most recently) SARS-CoV-2 [1-3]. During each epidemic, an accurate, rapid, and accessible molecular diagnostic test is highly essential for the control and prevention of viral diseases. In particular, a cluster of cases of pneumonia resulting from a new coronavirus, SARS-CoV-2, was initially described in late 2019 (or earlier); this disease was later named as COVID-19 [4]. During the epidemic, the Chinese government and people implemented approaches to aid in the diagnosis, isolation, and treatment of affected patients; they also strictly restricted the flow of people, which constituted the socalled "people's war". Therefore, the outbreak and spread of COVID-19 in China was largely contained after approximately 1.5 months. However, in most other countries, the disease has spread rapidly since late February 2020; on 12 March 2020, the World Health Organization recognized the COVID-19 as a global pandemic [5]. As of 30 August 2020, over 24 million confirmed cases and 838,924 deaths had been reported worldwide [6]. Thus far, effective specific drugs and vaccines specific for COVID-19 are unavailable; accordingly, rapid and accurate early detection of the COVID-19 causative virus (i.e.,

\footnotetext{
${ }^{1}$ Institute of Polymer Optoelectronic Materials and Devices, State Key Laboratory of Luminescent Materials and Devices, South China University of Technology, Guangzhou 510640, China

${ }^{2}$ Beijing National Laboratory for Molecular Sciences, State Key Laboratory for Structural Chemistry of Unstable and Stable Species, College of Chemistry and Molecular Engineering, Peking University, Beijing 100871, China

${ }^{3}$ Department of Energy and Resources Engineering, College of Engineering, Peking University, Beijing 100871, China

${ }^{4}$ Qingdao Shuojing Biological Technology Co., Ltd., Qingdao 266112, China

${ }^{5}$ Department of Pathology, Beijing Ditan Hospital Capital Medical University, Beijing 100015, China

${ }^{6}$ Institute of Digital Economy Industry, Hangzhou 310015, China

${ }^{7}$ Beijing Sylincom Technology Co., Ltd., Beijing 100081, China

${ }^{\dagger}$ These authors contributed equally to this work.

* Corresponding authors (emails: msfhuang@scut.edu.cn (Huang F); fmo@pku.edu.cn (Mo F); chinaroc001@126.com (Wang P); guoxf@pku.edu.cn (Guo X))
} 
SARS-CoV-2) is expected to aid in controlling the ongoing pandemic and support resumption of normal life and economic conditions.

SARS-CoV-2 mainly consists of a single-stranded RNA genome (approximately 30,000 nucleotides) and four structural proteins that include the spike surface glycoprotein (S), envelope protein (E), membrane protein $(\mathrm{M})$, and nucleocapsid protein $(\mathrm{N})$. There are generally two strategies for identification of the virus: (1) detection of viral RNA and (2) detection of host antibodies. Currently, the presence of specific viral RNA sequences in the patient samples is considered definitive proof of COVID-19, while immunodetection is regarded as a useful auxiliary technique. Reverse transcriptase quantitative polymerase chain reaction (RT-qPCR) is the primary method for detection of nucleic acid-based genetic sequences from any organism, including SARS-CoV-2 [7-11]. Because of the labor-intensive sample preparation, which must be conducted in a biological laboratory by professionals using specialized instruments, the typical turn-around time of the RT-qPCR method is longer than $24 \mathrm{~h}$. The complex sample preparation steps prior to testing might also reduce clinical sensitivity (the percentage of actual positive individuals identified as positive individuals), resulting in false negative results $[12,13]$. In addition, point-of-care detection is not accessible by the RT-qPCR method. Hence, the development of highly sensitive and specific nucleic acid testing methods, in combination with the characters of fewer sample preparation steps and the use of a portable detection instrument, is an extremely attractive prospect for accurate and rapid diagnosis of COVID-19.

Biosensors, as an alternative and reliable solution for the analysis of biomolecules, has been drawing considerable research interest [14-17]. Among different kinds of optical and electrical biosensing technologies currently avalaible, field-effect transisitor (FET)-based biosensors are particularly attractive because of their remarkable features, including no fluorescent labeling requirement, highly sensitive detection, mass-production capability and low cost. The types of the FET biosensors mainly include silicon nanowire FETs, graphene/carbon nanotube FETs, organic FETs, compound-semiconductor FETs and ion sensitive FETs [18-20]. Graphene materials possess excellent properties of large surface area, high electronic conductivity and high carrier mobility, making the graphene FET an ideal platform for biomolecule detection $[14,21]$.

In the present study, we developed an unprecedented accurate, rapid, and portable electrical detector based on the use of graphene FETs (G-FETs) for detection of RNA from COVID-19 patients. As shown in Fig. 1, the detection system mainly consists of two parts: a plug-andplay packaged biosensor chip and a home-developed electrical measurement machine. Each packaged chip contains ten G-FETs; specific ss-DNA probes are immobilized onto the graphene surface via $\pi-\pi$ stacking of a typical linker (1-pyrenebutyric acid N-hydroxysuccinimide ester, PBASE) [22-24]. The unique feature of this method is that the extent of hybridization between the ss-DNA probe and viral RNA can be directly converted to the current change of graphene channels without repetition of the PCR process. Our G-FET biosensors exhibited excellent performance for the detection of the RNA-dependent RNA polymerase (RdRp) gene target of SARS-CoV-2 with an ultra-low limit of detection (LOD) of $\sim 0.1 \mathrm{fg} \mathrm{mL}^{-1}$. Furthermore, we validated our method using clinical samples collected from ten patients with COVID-19 infection and eight healthy individuals, and the testing results were in full agreement with those of PCR-based optical methods. The entire process, precluding the extraction of detection targets from oropharyngeal swabs, requires approximately $10 \mathrm{~min}$. Because it does not involve time-consuming PCR step nor expensive instruments, our detection system has the potential to enable massive point-of-care testing of COVID19 , outside of specialized diagnostic laboratories, with the advantage of high sensitivity and low cost.

Notably, false negative results are inevitable in the course of nucleic acid testing; thus, the use of immunodetection as an auxiliary technique is important in the diagnosis of COVID-19 patients, especially those with suspected diseases [25-28]. By replacing the ss-DNA probe with a SARS-CoV-2 antigen protein, our detection system can also detect SARS-CoV-2 IgM and IgG antibodies with an ultra-low LOD of $\sim 1 \mathrm{fg} \mathrm{mL}^{-1}$. Immunoassays of serum specimens of six COVID-19 patients and three healthy subjects matched excellently with those of PCR-based optical methods.

\section{EXPERIMENTAL SECTION}

\section{Fabrication of graphene-based biosensor chips}

High-quality chemical vapor deposition (CVD)-grown monolayer graphene on 4 -inch silicon dioxide $(300 \mathrm{~nm})$ /Si wafer substrate was purchased from Jiangsu XFNANO Materials Tech Co., Ltd. The source/drain metal electrode arrays $(8 \mathrm{~nm} \mathrm{Cr} / 600 \mathrm{~nm} \mathrm{Au}$ ) were patterned by photolithography and thermal evaporation, followed by electron beam evaporation of 40 -nm-thick $\mathrm{SiO}_{2}$ to passivate 


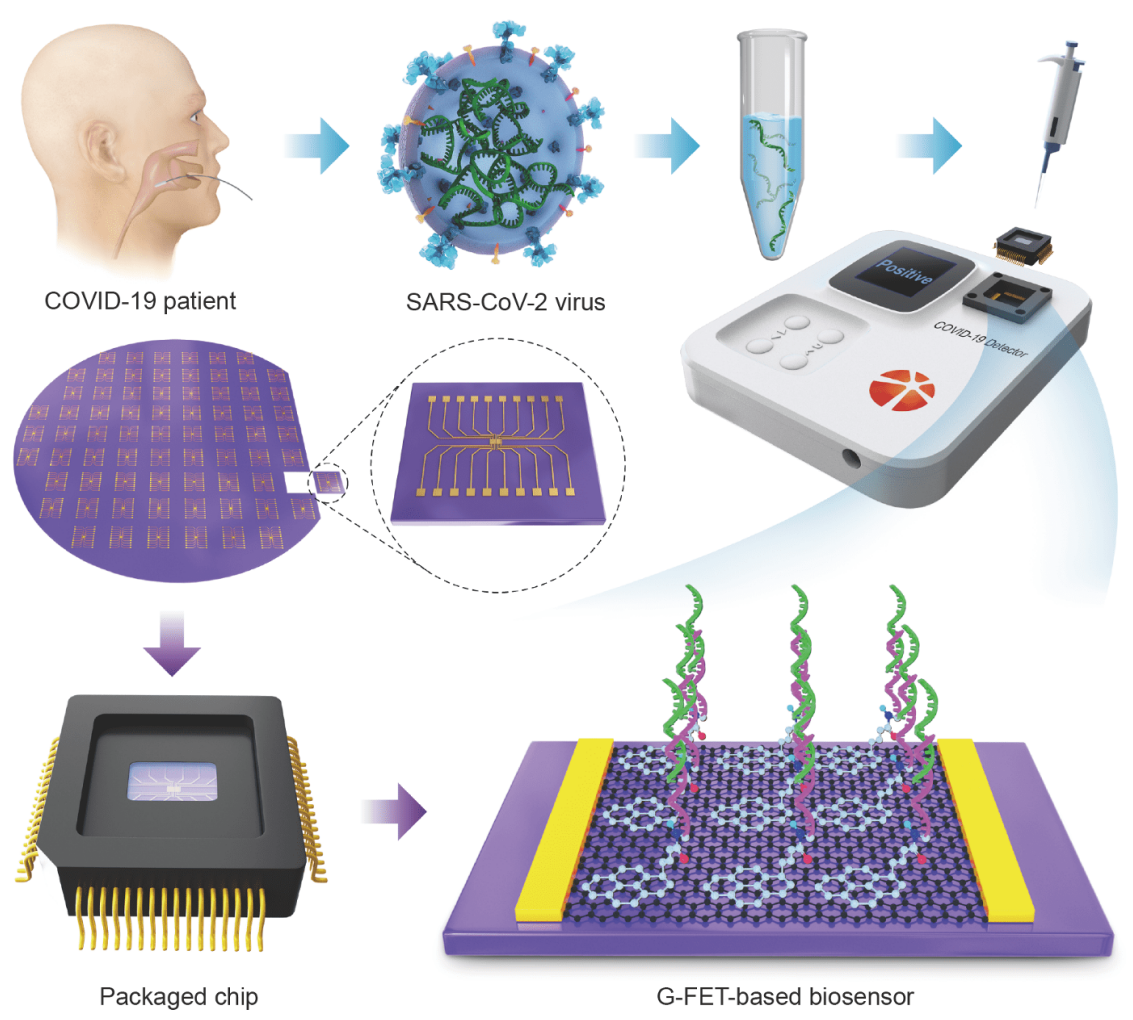

Figure 1 Schematic diagram of the operation procedure of our G-FET-based biosensing system for COVID-19. Top left: Extraction of viral RNA. Bottom left: from Si wafer to plug-and-play graphene packaged chips. Top right: home-developed portable electrical detector. Bottom right: illustration of the ss-DNA probe immobilization onto graphene using a typical PBASE linker, followed by hybridization with an RNA target.

the electrode surface (Fig. S1a, b).

Then, the 4-inch G-FET wafer was packaged into biosensor chips through Plastic Quad Flat Package (PQFP) in the following steps: (1) cut into approximately 425 small chips $(4 \mathrm{~mm} \times 4 \mathrm{~mm})$; (2) bond the chip with QFP64 ceramic package (Fig. S1c); (3) cover the contact point with glue to protect the electrical circuit.

\section{Modification of the graphene surface}

The freshly as-fabricated G-FET biosensor chips were immersed in $1 \mathrm{mmol} \mathrm{L}^{-1}$ PBASE (Sigma-Aldrich) in $\mathrm{CH}_{3} \mathrm{CN}$ at room temperature for $6 \mathrm{~h}$, then rinsed several times with $\mathrm{CH}_{3} \mathrm{CN}$ and dried using mild stream of $\mathrm{N}_{2}$ flow [22,23]. Subsequently, the PBASE-modified chips were exposed to either $10 \mu \mathrm{g} \mathrm{mL}^{-1}$ ss-DNA probe (Beijing RuiBiotech Co., Ltd.) or $100 \mu \mathrm{g} \mathrm{m}^{-1}$ antigen protein in PBS solution at room temperature overnight; they were then being dried by a mild stream of $\mathrm{N}_{2}$ gas, yielding ssDNA probe-immobilized chips and antigen protein-immobilized chips, respectively. The antigen protein employed in this study was a recombinant protein comprising the $\mathrm{S}$ and $\mathrm{N}$ protein of SARS-CoV-2, developed by Qingdao Hightop Biotech Co., Ltd. (For S protein, the receptor-binding domain (RBD) was chosen. The coincidence rates of $\mathrm{N}$ protein to those of SARS or MERS were $~ 78.12 \%$ and $\sim 39.67 \%$, respevtively. Although the coincidence rate bwteen SARS-CoV-2 and SARS is high, SARS has almost dissappeared. There are abuntant antigenic determinants in $\mathrm{N}$ protein with high specificity, which can avoid missing inspection. Therefore, the full length of $\mathrm{N}$ protein was chosen and recombined with the $\mathrm{RBD}$ domain of $\mathrm{S}$ protein.).

\section{Surface characterization and electrical measurements}

The morphology of the G-FET devices was determined by optical microscopy (Nikon Eclipse LV100 POL Microscope). Raman spectroscopy was performed on a confocal Raman spectrograph (JY Horiba HR800) in the backscattering geometry; the laser $(532 \mathrm{~nm})$ was focused on the sample by means of a $100 \times$ objective lens (numerical aperture $(\mathrm{NA})=0.9$ ). Analysis of the chemical binding component was determined by X-ray photoelectron 
spectroscopy (XPS, Kratos Analytical Ltd., AXIS Supra), with the incident beam produced by an $\mathrm{Al} \mathrm{X}$-ray source $(150 \mathrm{~W})$ and a pass energy of $160 \mathrm{eV}$. Surface roughness analysis was conducted by atomic force microscopy (AFM, Bruker, Dimension Icon with Nanoscope V controller). Electrical characterizations, including the transfer curves and current-voltage $\left(I_{\mathrm{D}}-V_{\mathrm{D}}\right)$ curves, were carefully carried out at room temperature using a Keysight B1500A semiconductor device analyzer (direct-current measurements) and a Karl Suss (PM5) manual probe station. IgG antibody protein was purchased from Beijing Sino Biological Inc. (isoelectrical point of $\sim 6.47$ and negetively charged at $\mathrm{pH}$ 7).

\section{Extraction of viral RNA and detection of clinical samples} RNA samples from both COVID-19 patients and healthy subjects were extracted from oropharyngeal swabs using QiAamp Viral RNA Mini Kits (Qiagene), which required approximately about $15 \mathrm{~min}$ for each sample. The extracted RNA solution $(25 \mu \mathrm{L})$ was directly added to the packaged chip surface, and heated at $85^{\circ} \mathrm{C}$ for $9 \mathrm{~min}$. Afterwards, the chip was gently washed with deionized (DI) water to remove the unbonded RNA and dried before measurements with our home-developed electrical detector (Fig. S2). Similarly, for immunoassays, each serum specimen $(25 \mu \mathrm{L})$ was added to the packaged chip surface at room temperature for $4 \mathrm{~min}$; the surface was rinsed with DI water and dried with a stream of $\mathrm{N}_{2}$ gas before electrical measurements were taken.

\section{RESULTS AND DISCUSSION}

\section{Surface characterization}

Fig. 2a displays an optical image of the ten G-FETs in one packaged biosensor chip. The dimensions of the sensing area are $40 \mu \mathrm{m} \times 8 \mu \mathrm{m}($ Length $\times$ Width $)$ for each G-FET. To avoid undesired adsorption of nucleic acids on the surface of gold electrodes and reduce interference during electrical measurements, the electrodes were protected by evaporation of an insulating layer of $40 \mathrm{~nm} \mathrm{SiO} 2$. Details of the fabrication process of a G-FET array and the packaging procedure of the chip are provided in the EXPERIMENTAL SECTION and Supplementary information. As displayed in Fig. 2b-d, Raman spectrum analysis and high-resolution XPS were performed to demonstrate that PBASE were efficiently modified onto the graphene surface. In the Raman spectrum of pristine graphene, two typical major peaks ( $G$ and $2 D$ peaks) were observed, which corresponded to the lattice vibration mode and second-order Raman scattering, respectively.
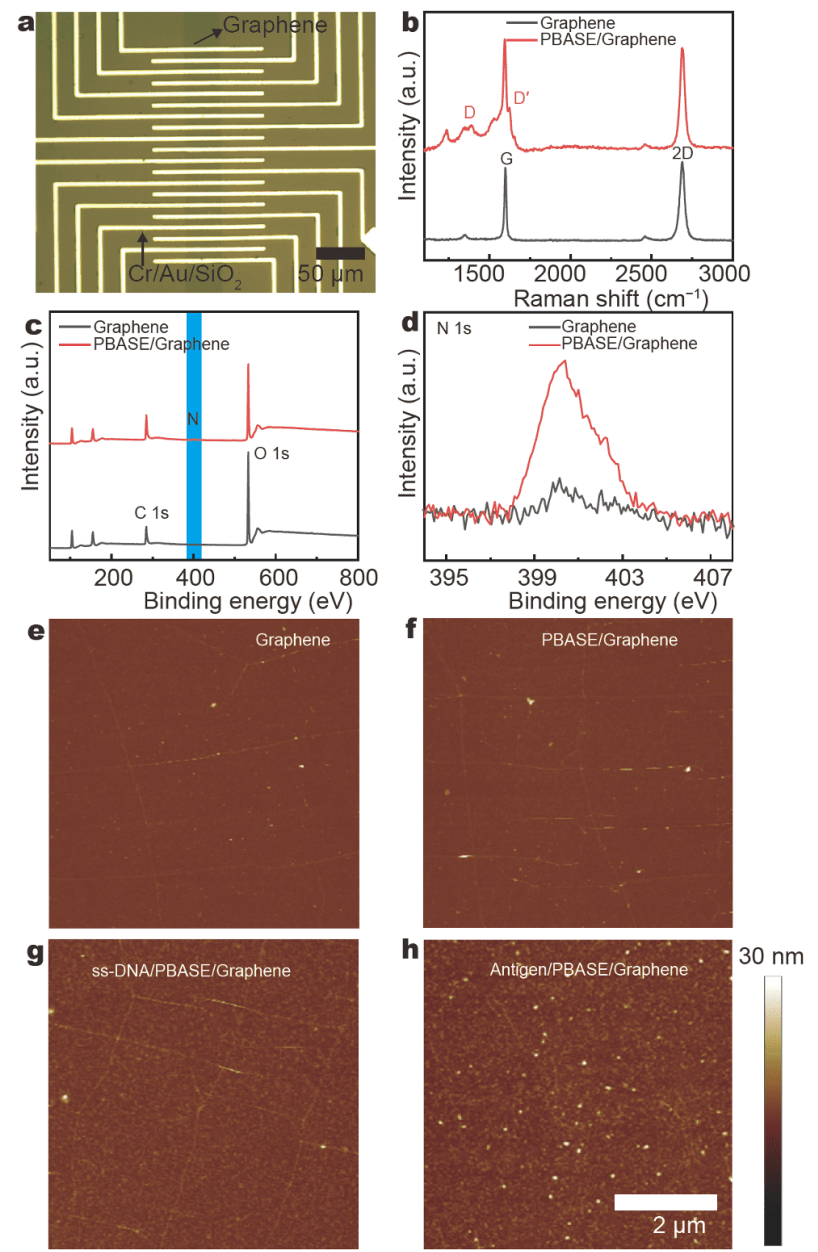

Figure 2 Surface analyses of pristine, PBASE-modified, ss-DNA-immobilized, and antigen-immobilized graphene using Raman spectroscopy, XPS, and AFM. (a) Optical image of a G-FET array in a packaged biosensor chip. (b) Raman spectra of pristine and PBASE-modified graphene. (c, d) High-resolution XPS spectra and enlarged N 1s region for pristine and PBASE-modified graphene. (e-h) AFM images of pristine graphene (e), PBASE-modified graphene (f), ss-DNA probeimmobilized graphene $(\mathrm{g})$ and antigen protein-immobilized graphene (h). Scale bar $=2 \mu \mathrm{m}$.

The single Lorentz type 2D peak and the high integrated peak area ratio value of $\sim 2.52$ between $2 \mathrm{D}$ and $\mathrm{G}$ peaks indicated high-quality monolayer graphene [29]. While in the Raman spectrum of PBASE-modified graphene, D and D' peaks apparently appeared, stemming from the binding between the pyrene group and graphene. Fig. $2 \mathrm{~d}$ shows the comparison of the $\mathrm{N} 1 \mathrm{~s}$ region before and after the PBASE functionalization of graphene, enlarged from the XPS spectrum (Fig. 2c). For pristine graphene, the $\mathrm{N}$ 1s peak is nearly absent; the obviously increased intensity of the $\mathrm{N}$ 1s peak after modification indicates successful immobilization of PBASE on the graphene 
surface.

We further exploited AFM to visualize the quality of surface functionalization. Fig. 2e-h are AFM images of pristine graphene, PBASE-modified graphene, ss-DNA probe-immobilized graphene, and antigen protein-immobilized graphene, respectively. The corresponding height profiles and surface roughness (RMS) of graphene are depicted in Fig. S3. After PBASE assembly, the RMS of graphene slightly increased from $\sim 0.21$ to $\sim 0.40 \mathrm{~nm}$, further reaching $\sim 0.77$ and $\sim 1.25 \mathrm{~nm}$ with following immobilization of the ss-DNA probe and antigen protein, respectively. The AFM results are in consistence with the larger antigen protein size, compared with the ss-DNA probe. These findings confirmed the successful modification of graphene.

\section{Electrical characterization}

Transfer curves of G-FETs were also measured to confirm efficient immobilization of the ss-DNA probe on the graphene surface and to monitor the specific interaction between the ss-DNA probe and its complementary gene sequence (i.e., RdRp target). Ionic liquid as the dielectric layer has been proven to constitute an effective strategy for modulation of charge transport in semiconductor devices $[30,31]$. Here, we employed ionic liquid containing $\quad \mathrm{N}, \mathrm{N}$-diethyl- $\mathrm{N}$-(2-methoxyethyl)- $N$-methylammonium bis(trifluoromethylsulfonyl)imide (DEMETFSI), to prepare an ionic liquid-gated FET (structure shown in Fig. S4a). For pristine graphene, the transfer curve (Fig. S4b, black line) exhibits the typical ambipolar character of graphene with the Dirac point localized at a gate voltage of $\sim 0.38 \mathrm{~V}$ ( $\left.V_{\text {Dirac }}\right)$. The positive value of $V_{\text {Dirac }}$ indicates that the graphene is $p$-doped, resulting from chemical doping by the residues of the processing chemicals; this phenomenon is common in CVD-grown graphene processed via wet transfer [32]. After immobilization of the ss-DNA probe and hybridization with the complementary RdRp target, the value of $V_{\text {Dirac }}$ shifted by $\sim 0.38$ and $\sim 0.31 \mathrm{~V}$, sequentially. These results are in consistence with findings reported in previous literatures $[33,34]$. The transfer curve measurements of the pristine, antigen protein-modified, and antibody-bound graphene show a similar tendency (Fig. S4c).

\section{Sensitivity and reusability characterizations}

To evaluate the performance of the as-fabricated G-FET biosensors, we measured the $I_{\mathrm{D}}-V_{\mathrm{D}}$ curves of ss-DNA probe-modified devices in response to various concentrations of fully complementary RdRp target (fragment of the RdRp gene sequence in SARS-CoV-2) in phosphate buffer saline (PBS), with $V_{\mathrm{D}}$ ranging from -50 to $50 \mathrm{mV}$ (Fig. 3a, inset is the partially enlarged image). The sequences of the ss-DNA probe, RdRp target, and mismatched DNA are shown in Table S1. An excellent linear relationship between $I_{\mathrm{D}}$ and $V_{\mathrm{D}}$ was observed over the entire $V_{\mathrm{D}}$ scanning range. Therefore, the values of the $I_{\mathrm{D}}$ change ratio, in comparison with the origin of ss-DNA probe-modified biosensors $\left(\Delta I_{\mathrm{D}} / I_{0}\right)$ at $V_{\mathrm{D}}=50 \mathrm{mV}$, was extracted and plotted as a function of the concentration of RdRp target, as displayed in Fig. 3b. As the concentration of RdRp target increased, the slope of the curve gradually decreased, such that it reached a close equilibrium between the hybridization and electrostatic repulsion of ss-DNA probe and RdRp target at high concentrations. For control experiments, the G-FET biosensor was exposed to different concentrations of a mismatched DNA chain of identical length of or to PBS solution, a slight reduction in the value of $\Delta I_{\mathrm{D}} / I_{0}$ was observed (Fig. S5), revealing that the ss-DNA probemodified biosensor was highly specific to its complementary RdRp target. Remarkably, our G-FET biosensor exhibited very high sensitivity with an LOD as low as $\sim 0.1 \mathrm{fg} \mathrm{mL}^{-1}$, which constituted approximately 1800 copies per $\mathrm{mL}$; this was comparable to the LOD of the RT-qPCR method. Notably, our electrical method does not require the amplification steps inherent to RT-qPCR; it can also directly measure the gene target, which reduces the delay for results and lowers the possibility of interference. To examine the reusability of the G-FETs biosensor, the ss-DNA probe-RdRp target hybridization and dehybridization processes were conducted sequentially three times, as shown in Fig. 3e. The values of $\Delta I_{\mathrm{D}} / I_{0}$ were $\sim 7.2 \%, \sim 5.4 \%$, and $\sim 4.4 \%$ for the first, second, and third cycles, respectively, indicating that the G-FET biosensor could be reused multiple times. The reusability of the devices will enable cost reduction for each test, which is of considerable importance to the future commercialization of our product.

In addition to nucleic acid detection, we investigated the performance of antigen protein-modified G-FET biosensors in dynamic response to the SARS-CoV-2specific IgG antibody protein. Similarly, the $I_{\mathrm{D}}$ value of the graphene channel increased gradually with increasing IgG antibody protein concentration; a good linear relationship was preserved with $V_{\mathrm{D}}$ ranging from -50 to $50 \mathrm{mV}$, thereby providing an excellent LOD of $\sim 1 \mathrm{fg} \mathrm{mL}^{-1}$ (Fig. 3c, d), which ranks the highest among all immunodetection techniques and at least three orders of magnitude higher than the immune colloidal gold technique $[25,28]$. Collectively, these results indicated that our 

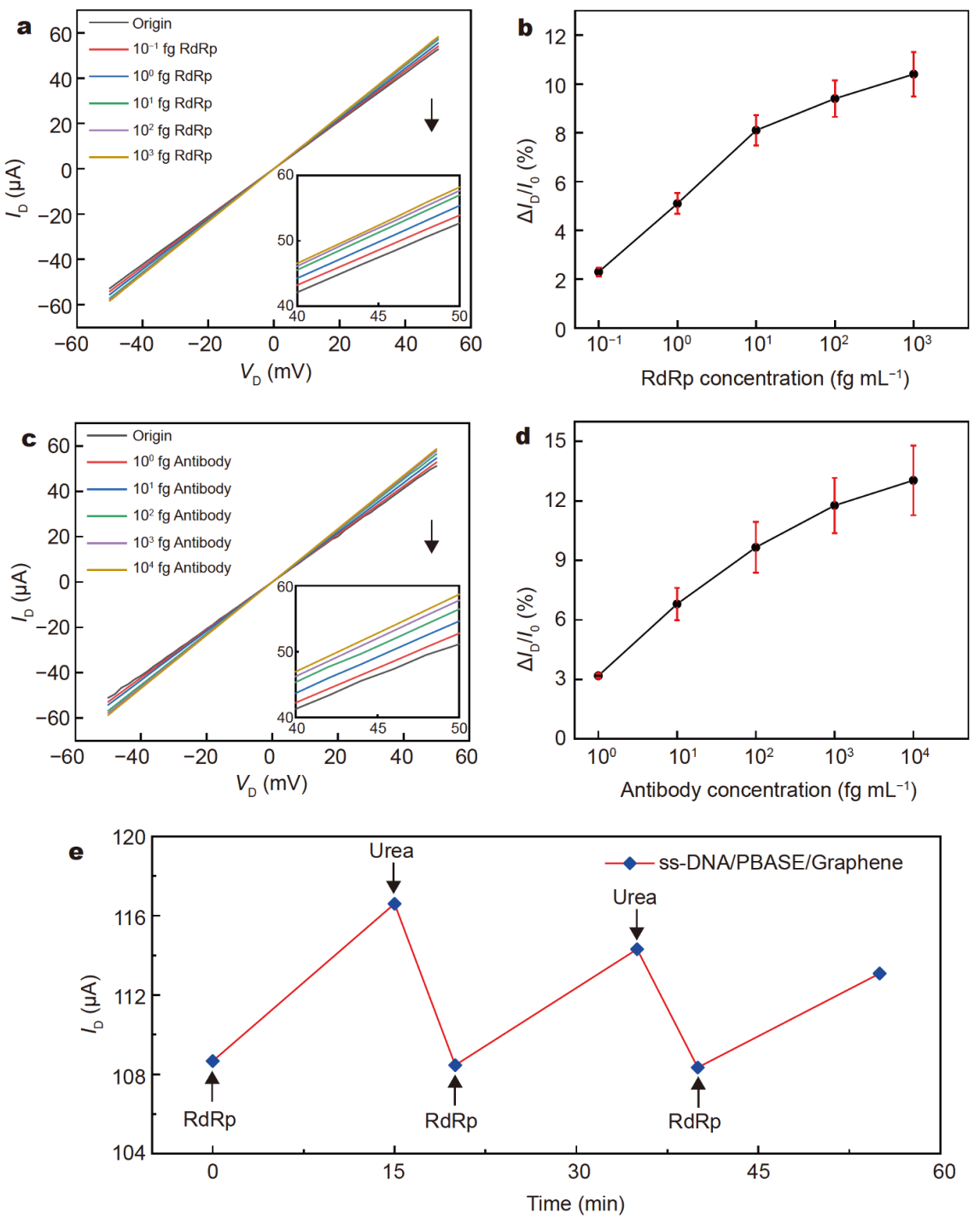

Figure 3 Sensitivity and reusability. (a, c) $I_{\mathrm{D}}-V_{\mathrm{D}}$ curves of G-FET biosensors responding to different concentrations of RdRp target (a) and antibody protein (c). Insets are partially enlarged curves. (b, d) Values of $\Delta I_{\mathrm{D}} / I_{0}$ at $V_{\mathrm{D}}=50 \mathrm{mV}$ extracted and plotted as a function of the concentration of RdRp target (b) and antibody protein (d). (e) Hybridization and dehybridization cycles of the same device.

G-FET biosensors have the potential to rapidly and sensitively detect both the gene RNA sequence of SARSCoV-2 and an IgG antibody protein specific for the virus, thus laying the foundation for the following clinical diagnostic assay.

\section{SARS-COV-2 detection from clinical samples}

Because our G-FET biosensors displayed excellent performance for detection of both SARS-CoV-2 RNA and SARS-CoV-2-specific IgG antibody protein in PBS, we tested their performance for detection of viral RNA in clinical samples, which were provided by Beijing Ditan Hospital Capital Medical University. To evaluate the performance of ss-DNA probe (RdRp gene) in detection of viral RNA in clinical samples, we firstly developed a DNA-RNA in-site hybridization (RNA-ISH) method to detect SARS-CoV-2 virus in tissue slides (Fig. S6). RNAISH is a non-amplification genetic test and a specific test due to long probe design (100 bps in our RNA-ISH system). Fluorescent image (Fig. S6b) of the bronchial brush specimen of COVID-19 patients clearly indicated effectiveness of the RdRp probe in detection of viral RNA. Afterwards, with ss-DNA probe 01, concentrationdependent measurements of viral RNA of clinical samples were perfomed, indicating an LOD of 1000 copies per mL (Fig. S7). For clinical detection, both COVID-19 patients 
and healthy subjects underwent sampling with oropharyngeal swabs, followed by the extraction of viral RNA. Then, the viral RNA was diluted by 100 folds before testing, without further sample processing. All tests of clinical samples were performed using the packaged biosensor chips in our home-developed portable COVID19 detector (Fig. S8). To achieve maximum accuracy, we designed and integrated ten G-FETs in each packaged biosensor chip. The resistance of ss-DNA probe-modified graphene was recorded as $R_{n}(n=1-10)$; the resistance was then recorded as $R_{n}^{\prime}$ after the addition of clinical samples from either COVID-19 patient or healthy subject. Subsequently, the value of the relative change in resistance $\left(R_{n}^{\prime}-R_{n}\right) / R_{n}$ of each graphene channel was calculated and averaged (defined as $\Delta R / R_{0}$ ) as the basis to determine whether a sample yielded positive or negative results. Table 1 summarizes the test results of ten COVID-19 patients and eight healthy subjects. The cutoff value of $\Delta R / R$ was set at -2 ; thus results with $\Delta R / R \leq-2$ were considered positive and those with $\Delta R / R>-2$ were considered negative. All ten COVID-19 patients were correctly identified as COVID-19 infected individuals while eight healthy individuals were correctly identified as healthy, indicating that our G-FET biosensors possess both high sensitivity and specificity in clinical diagnosis of COVID-19 patients. The detection process requires only $10 \mathrm{~min}$ after the extraction of viral RNA from oropharyngeal swabs (Fig. S9). In comparison with the RT-
qPCR approach, this method avoids the time-consuming step of viral RNA amplification, in combination with the home-developed portable detector. Future development of our portable detector will be focused on point-of-care testing outside of specialized diagnostic laboratories, which is of considerable importance for large-scale nucleic acid detection of COVID-19 and the ability of large populations to return to work and school.

As an auxiliary measure to complement nucleic acid detection of COVID-19, we assessed the efficiency of immunodetection by validating the performance of our method in the immunoassays of clinical samples. The serum specimens of six COVID-19 patients and three healthy subjects (provided by Beijing Ditan Hospital Capital Medical University) were diluted 100-fold before assessment using our G-FET biosensors. Analysis of each sample required approximately $5 \mathrm{~min}$. As summarized in Table 2, the testing results were in excellent agreement, indicating our biosensors provided accurate, rapid immunological diagnosis of COVID-19.

\section{CONCLUSIONS}

In summary, we developed an unprecedented reliable GFET-based detection system for convenient diagnosis of COVID-19, which consists of two parts: a plug-and-play packaged biosensor chip and a portable electrical measurement machine. This detecting system exhibits obvious advantages of high sensitivity, rapid speed $(\sim 10 \mathrm{~min}$

Table 1 Nucleic acid analysis of COVID-19 patients and healthy subjects ${ }^{\mathrm{a}}$

\begin{tabular}{cccccccccc}
\hline & Patient 1 & Patient 2 & Patient 3 & Patient 4 & Patient 5 & Patient 6 & Patient 7 & Patient 8 & Patient 9 \\
\hline$\Delta R / R_{0}(\%)$ & -9 & -5.8 & -2.8 & -3.6 & -5.9 & -8.9 & -6.2 & -3.1 & -6.1 \\
G-FET results & + & + & + & + & + & + & + & + & + \\
Agreement & Yes & Yes & Yes & Yes & Yes & Yes & Yes & Yes & Yes \\
& Patient 10 & Health 1 & Health 2 & Health 3 & Health 4 & Health 5 & Health 6 & Health 7 & Health 8 \\
$\Delta R / R_{0}(\%)$ & -5.4 & 1.6 & -0.3 & 2 & 3.2 & 4.1 & 5.3 & 2.9 & 2.2 \\
G-FET results & + & - & - & - & - & - & - & - & - \\
Agreement & Yes & Yes & Yes & Yes & Yes & Yes & Yes & Yes & Yes \\
\hline
\end{tabular}

a) "Cutoff value" was set at -2; "+" represents positive, and "-" represents negative; "Yes" indicates that the G-FET result is in consistence with the clinical standard samples.

Table 2 Antibody analysis of COVID-19 patients and healthy subjects ${ }^{\mathrm{a}}$

\begin{tabular}{cccccccccc}
\hline Number & Patient 1 & Patient 2 & Patient 3 & Patient 4 & Patient 5 & Patient 6 & Health 1 & Health 2 & Health 3 \\
\hline$\Delta R / R_{0}(\%)$ & -2.6 & -4.5 & -4.5 & -2.8 & -1.3 & -2.6 & 0.7 & 0.2 & 1.1 \\
G-FETs results & + & + & + & + & + & + & - & - & - \\
Agreement & Yes & Yes & Yes & Yes & Yes & Yes & Yes & Yes & Yes \\
\hline
\end{tabular}

a) "Cutoff value" was set at -1; "+" represents positive, and "-" represents negative; "Yes" indicates that the G-FET result is in consistence with the clinical standard samples. 
for RNA analysis and $\sim 5 \mathrm{~min}$ for immunoassay), and bifunction (both RNA analysis and immunoassay). These advantages enable high-throughput point-of-care testing, which may facilitate management of the current severe public health crisis. We are firmly of the opinion that this detection system offers a universal methodology that is ready for immediate application and rapid detection of various biomolecules and viruses, such as nucleic acids, proteins, biomarkers, SARS, swine flu, Ebola, and MERS.

Received 16 September 2020; accepted 25 November 2020; published online 29 December 2020

1 Zhu N, Zhang D, Wang W, et al. A novel coronavirus from patients with pneumonia in China, 2019. N Engl J Med, 2020, 382: 727-733

2 Wang C, Horby PW, Hayden FG, et al. A novel coronavirus outbreak of global health concern. Lancet, 2020, 395: 470-473

3 de Wit E, van Doremalen N, Falzarano D, et al. SARS and MERS: Recent insights into emerging coronaviruses. Nat Rev Microbiol, 2016, 14: 523-534

$4 \mathrm{Lu} \mathrm{R}$, Zhao X, Li J, et al. Genomic characterisation and epidemiology of 2019 novel coronavirus: Implications for virus origins and receptor binding. Lancet, 2020, 395: 565-574

5 WHO Coronavirus Disease 2019 (COVID-19): Situation Report -52. https://www.who.int/docs/default-source/coronaviruse/situation-reports/20200312-sitrep-52-covid-19.pdf?sfvrsn=e2bfc9c0_4

6 WHO Coronavirus Disease (COVID-19) Dashboard. https://covid19.who.int

7 Talebian S, Wallace GG, Schroeder A, et al. Nanotechnology-based disinfectants and sensors for SARS-CoV-2. Nat Nanotechnol, 2020, 15: 618-621

8 Broughton JP, Deng X, Yu G, et al. CRISPR-Cas12-based detection of SARS-CoV-2. Nat Biotechnol, 2020, 38: 870-874

9 Chan JFW, Yip CCY, To KKW, et al. Improved molecular diagnosis of COVID-19 by the novel, highly sensitive and specific COVID-19-RdRp/Hel real-time reverse transcription-PCR assay validated in vitro and with clinical specimens. J Clin Microbiol, 2020, 58: e00310-20

10 Real-time RT-PCR panel for detection 2019-nCoV (US Centers for Disease Control and Prevention, 2020). https://www.who.int/docs/ default-source/coronaviruse/uscdcrt-pcr-panel-for-detection-instructions.pdf

11 WHO Laboratory testing for coronavirus disease 2019 (Covid-19) in suspected human cases: Interim guidance, 2020. https://www. who.int/emergencies/diseases/novel-coronavirus-2019/technicalguidance/laboratory-guidance

12 Xie X, Zhong Z, Zhao W, et al. Chest CT for typical coronavirus disease 2019 (COVID-19) pneumonia: Relationship to negative RT-PCR testing. Radiology, 2020, 296: E41-E45

13 Zhang Q, Zhao Q. Inactivating porcine coronavirus before nuclei acid isolation with the temperature higher than $56^{\circ} \mathrm{C}$ damages its genome integrity seriously. BioRxiv, 2020, doi: 10.1101/ 2020.02.20.958785

$14 \mathrm{Lu} \mathrm{CH}$, Yang HH, Zhu CL, et al. A graphene platform for sensing biomolecules. Angew Chem Int Ed, 2009, 48: 4785-4787

$15 \mathrm{He} \mathrm{G}, \mathrm{Li} \mathrm{J}, \mathrm{Ci} \mathrm{H}$, et al. Direct measurement of single-molecule DNA hybridization dynamics with single-base resolution. Angew
Chem Int Ed, 2016, 55: 9036-9040

16 Ke G, Duan C, Huang F, et al. Electrical and spin switches in single-molecule junctions. InfoMat, 2020, 2: 92-112

$17 \mathrm{Su}$ D, Gu C, Guo X. Functional molecular electronic devices through environmental control. Sci China Mater, 2019, 62: 1-7

18 Syu YC, Hsu WE, Lin CT. Review-Field-effect transistor biosensing: Devices and clinical applications. ECS J Solid State Sci Technol, 2018, 7: Q3196-Q3207

19 Liang Y, Xiao M, Wu D, et al. Wafer-scale uniform carbon nanotube transistors for ultrasensitive and label-free detection of disease biomarkers. ACS Nano, 2020, 14: 8866-8874

20 Namdari P, Daraee H, Eatemadi A. Recent advances in silicon nanowire biosensors: Synthesis methods, properties, and applications. Nanoscale Res Lett, 2016, 11: 406

21 Szunerits S, Boukherroub R. Graphene-based biosensors. Interface Focus, 2018, 8: 20160132

22 Jia $\mathrm{C}, \mathrm{Li} \mathrm{H}$, Jiang J, et al. Interface-engineered bistable [2] rotaxanegraphene hybrids with logic capabilities. Adv Mater, 2013, 25: 6752-6759

23 Campos R, Borme J, Guerreiro JR, et al. Attomolar label-free detection of DNA hybridization with electrolyte-gated graphene field-effect transistors. ACS Sens, 2019, 4: 286-293

24 Seo G, Lee G, Kim MJ, et al. Rapid detection of COVID-19 causative virus (SARS-CoV-2) in human nasopharyngeal swab specimens using field-effect transistor-based biosensor. ACS Nano, 2020, 14: 5135-5142

25 Van Elslande J, Houben E, Depypere M, et al. Diagnostic performance of seven rapid IgG/IgM antibody tests and the Euroimmun IgA/IgG ELISA in COVID-19 patients. Clin Microbiol Infection, 2020, 26: 1082-1087

26 Tuaillon E, Bolloré K, Pisoni A, et al. Detection of SARS-CoV-2 antibodies using commercial assays and seroconversion patterns in hospitalized patients. J Infection, 2020, 81: e39-e45

27 Li Z, Yi Y, Luo X, et al. Development and clinical application of a rapid IgM-IgG combined antibody test for SARS-CoV-2 infection diagnosis. J Med Virol, 2020, 92: 1518-1524

28 Hou H, Wang T, Zhang B, et al. Detection of IgM and IgG antibodies in patients with coronavirus disease 2019. Clin Transl Immunol, 2020, 9: e1136

29 Malard LM, Pimenta MA, Dresselhaus G, et al. Raman spectroscopy in graphene. Phys Rep, 2009, 473: 51-87

30 Ye J, Craciun MF, Koshino M, et al. Accessing the transport properties of graphene and its multilayers at high carrier density. Proc Natl Acad Sci USA, 2011, 108: 13002-13006

31 Xin N, Li X, Jia C, et al. Tuning charge transport in aromatic-ring single-molecule junctions via ionic-liquid gating. Angew Chem Int Ed, 2018, 57: 14026-14031

32 Chen JH, Ishigami M, Jang C, et al. Printed graphene circuits. Adv Mater, 2007, 19: 3623-3627

33 Xu S, Zhan J, Man B, et al. Real-time reliable determination of binding kinetics of DNA hybridization using a multi-channel graphene biosensor. Nat Commun, 2017, 8: 14902

34 Cai B, Wang S, Huang L, et al. Ultrasensitive label-free detection of PNA-DNA hybridization by reduced graphene oxide field-effect transistor biosensor. ACS Nano, 2014, 8: 2632-2638

Acknowledgements This work was supported by the National Key R\&D Program of China (2017YFA0204901), the National Natural Science Foundation of China (21727806, 21772003 and 21933001), the Tencent Foundation through the XPLORER PRIZE, Guangdong Major 
Project of Basic and Applied Basic Research (2019B030302007), and Beijing National Laboratory for Molecular Sciences (BNLMS201901).

Author contributions Guo X, Mo F, Wang P and Huang F conceived and designed the experiments; Ke G, Su D and Li Y fabricated the devices and performed the device measurements; Zhao Y, Wang H, Xiao $\mathrm{F}$ and Yuan $\mathrm{Y}$ designed and built the measurement machines; Liu $\mathrm{W}$ and Yang $\mathrm{Z}$ provided the antigen protein; $\mathrm{Li} \mathrm{M}$ and Wang $\mathrm{P}$ provided the clinical samples; Guo X, Mo F, Wang P, Ke G and Su D analyzed the data and wrote the paper. All authors discussed the results and commented on the manuscript.

Conflict of interest The authors declare that they have no conflict of interest.

Supplementary Information Experimental details and supporting data are available in the online version of this paper.

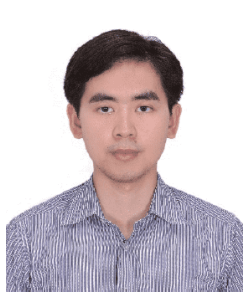

Guojun Ke received his BS degree in 2012 and $\mathrm{PhD}$ degree in 2017 from the School of Chemistry, Sun Yat-Sen University, respectively. From 2013 to 2016, he was a visiting student at the University of Basel. He is currently working as a postdoctoral fellow in South China University of Technology. His current research focuses on device physics of single-molecule junctions.

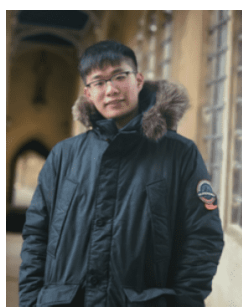

Dingkai Su received his BS degree in 2017 from the College of Nano Science and Technology, Soochow University. $\mathrm{He}$ is currently a $\mathrm{PhD}$ candidate at the College of Chemistry and Molecular Engineering, Peking University, under the guidance of Prof. Xuefeng Guo. His research interest focuses on single-molecule devices and dynamics.

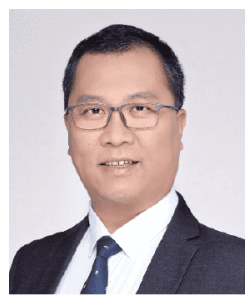

Xuefeng Guo received his $\mathrm{PhD}$ degree in 2004 from the Institute of Chemistry, Chinese Academy of Sciences. From 2004 to 2007, he was a postdoctoral research scientist at the Columbia University Nanocenter. He joined the faculty as a professor under the "Peking 100-Talent" Program at Peking University in 2008. His research focuses on functional nanometer/molecular devices.

\section{一种针对新型冠状病毒肺炎的准确、快速、便携 式双功能电检测仪}

柯国骏 ${ }^{1,2 \dagger}$, 苏鼎凯 ${ }^{2 \dagger}$, 李渝 ${ }^{2 \dagger}$, 赵钜 ${ }^{3}$, 王宏刚 ${ }^{3}$, 刘万键 ${ }^{4}$, 李慢 ${ }^{5}$, 杨志廷 ${ }^{4}$, 肖放 ${ }^{6}$, 袁尧 ${ }^{7}$, 黄飞 ${ }^{1 *}$, 莫凡洋 ${ }^{3 *}$, 王鹏 ${ }^{5^{*}}$, 郭雪峰 ${ }^{2^{*}}$

摘要 新型冠状病毒肺炎(COVID-19)正在多个国家快速传播, 已 经导致了严重的全球大流行. 由于目前没有针对此类病人的特效 药和针对此病毒的疫苗, 准确、快速地进行新冠病人检测成为了 控制大流行最有效的措施. 本文中我们开发了一种基于石墨烯场 效应晶体管的便携式双功能电检测仪，其通过核酸互补杂交或者 抗原-抗体特异性结合作用, 能分别进行病毒核酸序列检测和抗体 检测, 检测限分别低至 0.1 和 $1 \mathrm{fg} \mathrm{mL}^{-1}$. 我们通过临床样品检测进一 步评估了此方法：从 10 个新冠病人和 8 个正常人咽拭子中提取 RNA 直接用于核酸检测; 从 6 个新冠病人和 3 个正常人血清中提取抗体 用于抗体检测. 临床样品检测结果和基于聚合酶链反应的光学方 法结果完全吻合, 同时此方法拥有超快的检测速度(核酸检测需 $10 \mathrm{~min}$, 抗体检测需 $5 \mathrm{~min}$ ). 因此, 我们的实验提供了一种有效、准 确、高通量的新冠现场即时检测工具. 